\title{
WAP Based Seamless Roaming In Urban Environment with Wise Handoff Technique
}

\author{
N.S.V.Shet ${ }^{1}$, Prof.K.Chandrasekaran ${ }^{2}$ and Prof. K.C.Shet ${ }^{3}$ \\ NITK,Surathkal \\ shekar_shet@yahoo.com
}

\begin{abstract}
This paper highlights the studies performed to arrive at a novel handoff scheme in achieving uninterrupted connectivity. Every wireless communication user prefers seamless mobility and uninterrupted communication. Achieving this scenario is quite challenging both technologically and economically. Few of the reasons are geographical and man made constraints. Dynamic changes in environment and ever changing geographical landscape also pose serious difficulties to engineers involved in design of wireless systems. The following schemes have been suggested in an effort to improve Q.o.S of wireless mobile communication scenarios.
\end{abstract}

\section{Introduction}

From the days of cave men, man has always felt the need to communicate. Smoke was one means of communication and signaling, used by them. Today we are not satisfied with the reality of wireless videophones and wireless television on the move. We crave for virtual reality, which will be a boon for e-commerce. Buying products after seeing, feeling, smelling, tasting is what every consumer would prefer. However realizing all these is challenging and wireless communication is absolutely essential. Handoff becomes even more important due to the fact that the world is not flat and high frequency electromagnetic waves prefer traveling in straight lines. Low frequency electromagnetic waves fade easily with distance. Distances covered by man-made electromagnetic waves are not infinite. Seamless mobility is today's requirement since man has become highly mobile crossing oceans. Even within a premises man is mobile which is the cause for interrupted communication. Efficient handoff algorithms are the need of the hour to reduce infrastructure costs, save energy and improve QoS. This work is an attempt to achieve all these efficiently. Today's wireless users expect great things from tomorrow's wireless networks. These expectations have been fueled by hype about what the next generations of wireless networks will offer. The rapid increase of wireless subscribers increases the quality of services anytime, anywhere, and by any-media becoming indispensable. The necessity to merge or split cells in a cellular system arises due to the fact that, call traffic and user density in a geographical location is a dynamically changing phenomenon. It is also dependent on external factors such as time of the day, natural events, user behavior etc. User mobility is the main cause for variation in these two parameters. When the user density/population is rising, it becomes even more appropriate to make decisions about cell merging and splitting. Several techniques have evolved over time to address solutions, to these issues. An attempt to demonstrate the possibility of automatically merging or splitting cells, depending on parameters like call traffic density, user density, time of the day and channel resource availability has been tried by us earlier. [1]

Today wireless access points (WAP) are installed in many offices and public places. These WAP's can be used to provide the much needed connectivity at places where base station signals are difficult to reach. Scenarios were created and tested in our earlier work.[2] 


\section{Handoff}

The following paragraph present various aspects of handoff and discusses handoff related features of cellular systems. Desirable features of handoff are highlighted, and complexities of handoff are described. Several system deployment scenarios that dictate specific handoff requirements are illustrated. An account of handoff related resource management tasks of cellular systems is given. Implementation of the handoff process is explained. Some of the terminology used in cellular communications is explained next [1]

- Mobile Station (MS): The mobile station is intended for use while in motion at an unspecified location.

- Base Station (BS): The base station is a fixed station used for radio communication with Mobile Stations.

- Mobile Switching Center (MSC): The mobile switching center coordinates the routing of calls in a large service area. It is also referred to as the Mobile Telephone Switching Office (MTSO).

- Forward Channel: The forward channel is the radio channel used for the transmission of information from the base station to the mobile station. It is also known as the downlink.

- Reverse Channel: The reverse channel is the radio channel used for the transmission of information from the mobile station to the base station. It is also known as the uplink.

- Handoff (Handover): Handoff is a process of transferring a mobile station from one base station or channel to another. The channel change due to handoff occurs through a time slot for time division multiple access (TDMA), frequency band for frequency division multiple access (FDMA), and codeword for code division multiple access (CDMA) systems.

- Co-channel Interference (CCI): The co-channel interference is caused when the desired signal and another signal in some remote cell are using the same frequency or channel.

The following phases are involved in the planning of cellular communications:

\section{Assessment of Traffic Density}

- Determination of cell sizes and capacity;

- Decisions about Omni directional or sectored cells and antenna directions;

- Selection of best BS sites to cover the required area;

- Frequency allocation;

- Choice of power control parameters and selection of handoff parameters.

Let us now go into an in-depth investigation of the handoff aspects of cellular planning. The handoff process determines the spectral efficiency (i.e., the maximum number of calls that can be served in a given area) and the quality perceived by users. An efficient handoff algorithm effectively preserves and enhances the capacity and Quality of Service (QoS) of communication systems.

Figure 1 shows a simple handoff scenario in which an MS travels from BS A to BS B. Initially, the MS is connected to BS A. The overlap between the two cells is the handoff region in which the mobile may be connected to either BS A or BS B. At a certain time during the travel, the mobile is handed over from BS A to BS B. When the MS is close to BS B, it remains connected to BS B.

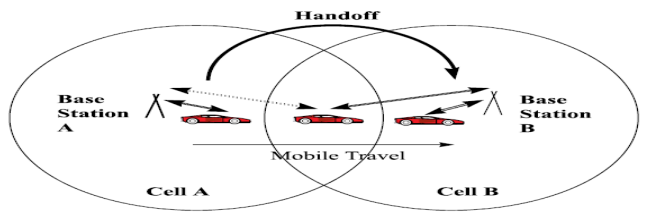

Fig .1: Handoff scenario in Cellular Communication 
The overall handoff procedure can be thought of as having two distinct phases: the initiation phase (in which the decision about handoff is made) and the execution phase (in which either a new channel is assigned to the MS or the call is forced to terminate). When a mobile moves from one base station to another, the handoff will occur. The received signal strength at mobile station is less than the threshold the handoffs will occur. If size of cell site is large then chances of handoff is less but it may occur if traffic is high.

In first generation mobile communication handoff criteria is based on signal strength and carrier to interference ratio.

Handoff based on Received Signal Strength: The

received signal strength is given by

$$
\mathrm{RSS}=\mathrm{C}+\mathrm{I} \text {. }
$$

Here threshold is fixed for RSS. If I is very large, then RSS is large, but handoff does not takes place, but it is needed. If I is very less, RSS is low, handoff takes place but it not needed.

Handoff based on Carrier to Interference Ratio: The $\mathrm{C} / \mathrm{I}$ ratio is given by

$$
(\mathrm{C}+\mathrm{I}) / \mathrm{I}=\mathrm{C} / \mathrm{I}
$$

Hand off dependent on $\mathrm{C} / \mathrm{I}$ ratio, $\mathrm{C} / \mathrm{I}$ drops if distance or interference increases. Here I measured before calls connect and $\mathrm{C}+\mathrm{I}$ is measured after call connect.

\section{Types of Handoff}

1. Intra system handoff.

2. Inter system handoff.

\section{Intra System Handoffs}

If handoff takes place in same wireless network it is called intra system handoffs. Intra system handoff is also called horizontal handoffs.

\section{Inter System Handoffs}

If handoff takes place in different wireless networks it is called inter system handoffs. It is also called as vertical handoffs.

\section{Handoff Methods}

1. Hard Handoffs.

2. Soft Handoffs.

\section{Hard Handoffs (Hard handoff is a break before make type connection):}

In hard handoff, the old connection breaks before the user transfer to the new channel. The hard handoffs are used in Time Division Multiple Access (TDMA) and Frequency Division Multiple Access (FDMA).

\section{Soft Handoffs (Soft handoff is a make before break connection):}

In Soft handoff, the mobile station measures the old as well as available signal strength, if the old signal has sufficient strength, call continues with old channel otherwise breaks the old channel and connects to new channel. Soft handoffs are used in Code Division multiple access systems (CDMA). 


\section{Handoff Initiation:}

A hard handoff occurs when the old connection is broken before a new connection is activated. The performance evaluation of a hard handoff is based on various initiation criteria. It is assumed that the signal is averaged over time, so that rapid fluctuations due to the multi-path nature of the radio environment can be eliminated. Numerous studies have been done to determine the shape as well as the length of the averaging window and the older measurements may be unreliable. Figure 2 shows a MS moving from one BS (BS1) to another (BS2). The mean signal strength of BS1 decreases as the MS moves away from it. Similarly, the mean signal strength of BS2 increases as the MS approaches it. This figure is used to explain various approaches described in the following subsection.

Handoff initiation is based on

1. Relative signal strength

2. Relative Signal Strength with Threshold

3. Relative Signal Strength with Hysteresis

4. Relative Signal Strength with Hysteresis and Threshold

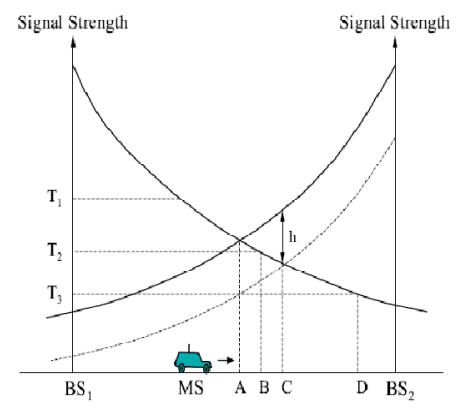

Fig 2: Signal strength and hysteresis between two adjacent BSs for potential handoff

\section{Relative Signal Strength}

This method selects the strongest received BS at all times. The decision is based on a mean measurement of the received signal. In Figure 4.2 the handoff would occur at position A. This method is observed to provoke too many unnecessary handoffs, even when the signal of the current BS is still at an acceptable level.

\section{Relative Signal Strength with Threshold}

This method allows a MS to hand off only if the current signal is sufficiently weak (less than threshold) and the other is the stronger of the two. The effect of the threshold depends on its relative value as compared to the signal strengths of the two BSs at the point at which they are equal. If the threshold is higher than this value, say $T 1$ in Figure 2, this scheme performs exactly like the relative signal strength scheme, so the handoff occurs at position A. If the threshold is lower than this value, say $T 2$ in Figure 2, the MS would delay handoff until the current signal level crosses the threshold at position B. In the case of $T 3$, the delay may be so long that the MS drifts too far into the new cell. This reduces the quality of the communication link from BS1 and may result in a dropped call. In addition, this results in additional interference to co-channel users. Thus, this scheme may create overlapping cell coverage areas. A threshold is not used alone in actual practice because its effectiveness depends on prior knowledge of the crossover signal strength between the current and candidate B S's. 


\section{Relative Signal Strength with Hysteresis}

This scheme allows a user to hand off only if the new BS is sufficiently stronger (by a hysteresis margin, $h$ in Figure 2) than the current one. In this case, the handoff would occur at point $C$. This technique prevents the so-called ping-pong effect, the repeated handoff between two BSs caused by rapid fluctuations in the received signal strengths from both BSs. The first handoff, however, may be unnecessary if the serving BS is sufficiently strong.

\section{Relative Signal Strength with Hysteresis and Threshold}

This scheme hands a MS over to a new BS only if the current signal level drops below a threshold and the target BS is stronger than the current one by a given hysteresis margin. In Figure 2, the handoff would occur at point $\mathrm{D}$ if the threshold is $T 3$.

\section{Handoff Decision}

There are numerous methods for performing handoff, at least as many as the kinds of state information that have been defined for MSs, as well as the kinds of network entities that maintain the state information. The decision-making process of handoff may be centralized or decentralized (i.e., the handoff decision may be made at the MS or network). From the decision process point of view, one can find at least three different kinds of handoff decisions.

Handoff decision types

1. Network Controlled Handoff

2. Mobile Assisted Handoff

3. Mobile controlled Handoff

\section{Network-Controlled Handoff}

In a network-controlled handoff protocol, the network makes a handoff decision based on the measurements of the MSs at a number of BSs. In general, the handoff process (including data transmission, channel switching, and network switching) takes 100 to 200ms. Information about the signal quality for all users is available at a single point in the network that facilitates appropriate resource allocation. Network-controlled handoff is used in first-generation analog systems such as AMPS (advanced mobile phone system) TACS (total access communication system), and NMT (advanced mobile phone system).

\section{Mobile-Assisted Handoff}

In a mobile-assisted handoff process, the MS makes measurements and the network makes the decision. In the circuit-switched GSM (global system mobile), the BS controller (BSC) is in charge of the radio interface management. This mainly means allocation and release of radio channels and handoff management. The handoff time between handoff decision and execution in such a circuit-switched GSM is approximately 1 second.

\section{Mobile-Controlled Handoff}

In mobile-controlled handoff, each MS is completely in control of the handoff process. This type of handoff has a short reaction time (of the order of 0.1 second). MS measures the signal strengths from surrounding BSs and interference levels on all channels. A handoff can be initiated if the signal strength of the serving BS is lower than that of another BS by a certain threshold. 


\section{Wireless Access Point}

A wireless access point (WAP) is a device that allows wireless communication devices to connect to a wireless network using Wi-Fi, Bluetooth or related standards. The WAP usually connects to a wired network, and can relay data between the wireless devices (such as computers or printers) and wired devices on the network.

\section{Common Applications of Wireless Access Point}

A typical corporate use, involves attaching several WAP's to a wired network and then providing wireless access to the office LAN. The wireless access points are managed by a WLAN (Wireless LAN) Controller which handles automatic adjustments to RF power, channels, authentication, and security. Further, controllers can be combined to form a wireless mobility group to allow inter-controller roaming. The controllers can be part of a mobility domain to allow clients access throughout large or regional office locations. This saves the client's time and administrators overhead because it can automatically re-associate or re-authenticate. Further, multiple controllers and all of the hundreds of access points attached to those controllers can be managed by a software called Cisco Wireless Control System which handles the same functions as a controller yet adds the bonus features of mapping user or RFID locations to an uploaded map, upgrading controllers and access point firmware, and rogue detection/handling. In this instance, the WAP functions as a wireless gateway for clients to access the wired network.

A Hot Spot is a common public application of WAP's, where wireless clients can connect to the Internet without regard for the particular networks to which they have attached for the moment. The concept has become common in large cities, where a combination of coffeehouses, libraries, as well as privately owned open access points, allow clients to stay more or less continuously connected to the Internet, while moving around. A collection of connected Hot Spots can be referred to as a lily-pad network. The majority of WAP's are used in Home wireless networks. Home networks generally have only one WAP to connect all the computers in a home. Most are wireless routers, meaning converged devices that include the WAP, a router, and, often, an Ethernet switch. Ethernet switch may also include a broadband modem.

\section{Limitations of Wireless Access Points}

One IEEE 802.11 WAP can typically communicate with 30 client systems located within a radius of $100 \mathrm{~m}$. However, the actual range of communication can vary significantly, depending on such variables as indoor or outdoor placement, height above ground, nearby obstructions, other electronic devices that might actively interfere with the signal by broadcasting on the same frequency, type of antenna, the current weather, operating radio frequency, and the power output of devices. Network designers can extend the range of WAP's through the use of repeaters and reflectors, which can bounce or amplify radio signals that ordinarily would go un-received. In experimental conditions, wireless networking has operated over distances of several kilometers. Most jurisdictions have only a limited number of frequencies legally available for use by wireless networks. Usually, adjacent WAP's will use different frequencies (Channels) to communicate with their clients in order to avoid interference between the two nearby systems. Wireless devices can "listen" for data traffic on other frequencies, and can rapidly switch from one frequency to another to achieve better reception. However, the limited number of frequencies becomes problematic in crowded downtown areas with tall buildings using multiple WAP's. In such an environment, signal overlap becomes an issue causing interference, which results in signal drop-age and data errors.

Wireless networking lags behind wired networking in terms of increasing bandwidth and throughput. While typical wireless devices for the consumer market can reach speeds of $11 \mathrm{Mbps}$ (megabits per second) (IEEE 802.11b) or $54 \mathrm{MB} / \mathrm{s}$ IEEE 802.11a, IEEE 802.11g), wired 
hardware of similar cost reaches $1000 \mathrm{Mbps}$ (Gigabit Ethernet). One impediment to increasing the speed of wireless communications comes from Wi-Fi's use of a shared communications medium, so a WAP is only able to use somewhat less than half the actual over-the-air rate for data throughput. Thus a typical $54 \mathrm{Mbps}$ wireless connection actually carries TCP/IP (Transmission Control Protocol/Internet Protocol) data at 20 to $25 \mathrm{Mbps}$. Users of legacy wired networks expect faster speeds, and people using wireless connections keenly want to see the wireless networks catch up. As of 2007 a new standard for wireless, 802.11n is awaiting final certification from IEEE. This new standard operates at speeds up to $540 \mathrm{Mbps}$ and at longer distances $(\sim 50 \mathrm{~m})$ than $802.11 \mathrm{~g}$. Use of legacy wired networks (especially in consumer applications) is expected to decline sharply as the common $100 \mathrm{Mbps}$ speed is surpassed and users no longer need to worry about running wires to attain high bandwidth.

\section{Advantages with WAP Handoff Techniques}

1) Uninterrupted connectivity at all places especially in urban environment.

2) Reduced load on base station processing.

3) Low signal strength requirement for Mobile stations.

4) Ease of system design

5) Low power at MS means longer battery discharge life

Simulation scenes created

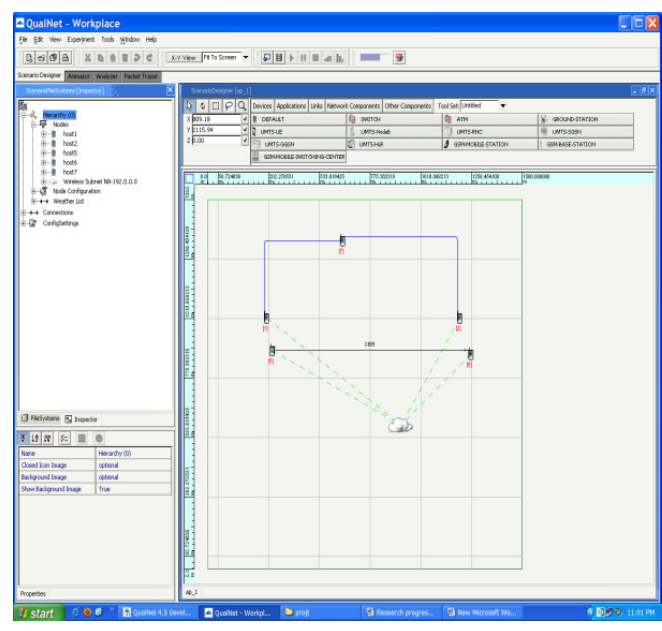

Fig a: initial scenario

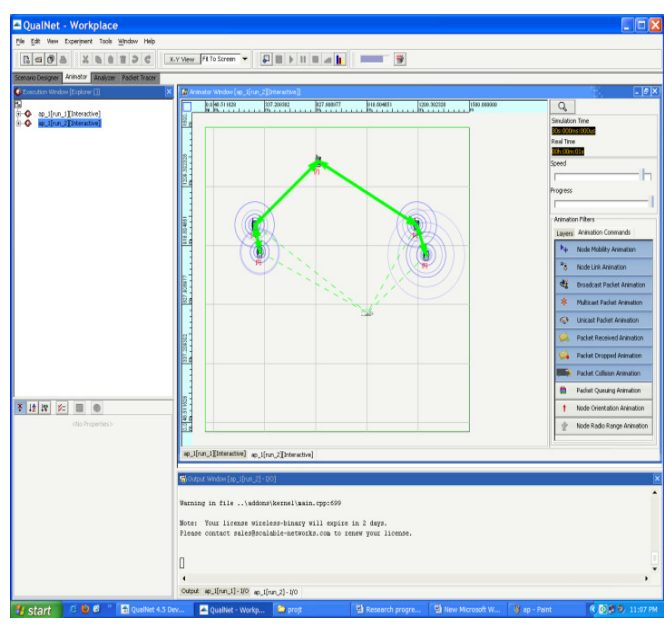

Fig b: final scenario during simulation.

The scene consists of two base stations, two WAPs, two mobile stations and one Mobile switching centre. The scene is designed such that the two mobile stations which are initially near two different base stations. Hear both mobile stations are in coverage area, mobility is there. 
Comparison table of simulation results obtained for wireless access points technique

\begin{tabular}{|l|l|l|l|l|l|}
\hline & $\begin{array}{l}\text { Scena } \\
\text { rio 1 }\end{array}$ & $\begin{array}{l}\text { Scena } \\
\text { rio 2 }\end{array}$ & $\begin{array}{l}\text { Scena } \\
\text { rio 3 }\end{array}$ & $\begin{array}{l}\text { Scena } \\
\text { rio 4 }\end{array}$ & $\begin{array}{l}\text { Scenario } \\
5\end{array}$ \\
\hline No of BS's & 2 & 2 & 2 & 2 & 4 \\
\hline No of MS's & 2 & 2 & 2 & 2 & 8 \\
\hline Mobility & NO & Yes & Yes & Yes & yes \\
\hline $\begin{array}{l}\text { No of mov } \\
\text { MS's }\end{array}$ & 0 & 1 & 1 & 2 & 3 \\
\hline No of WAP & 0 & 0 & 1 & 2 & 4 \\
\hline $\begin{array}{l}\text { CBR client } \\
\text { pack snt }\end{array}$ & 24 & 24 & 24 & 24 & 96 \\
\hline $\begin{array}{l}\text { CBR client } \\
\text { thrup(bits/s) }\end{array}$ & $4.24 *$ & $4.24 *$ & $4.24 *$ & $4.24 *$ & $4.24 * 10^{*}$ \\
\hline $\begin{array}{l}\text { CBR Server } \\
\text { pack recd }\end{array}$ & 24 & 8 & 24 & 24 & 80 \\
\hline $\begin{array}{l}\text { CBR Server } \\
\text { thrup(bits/s) }\end{array}$ & $4.24 *$ & $1.15 *$ & $4.24 *$ & $4.24 *$ & $2.15^{*} 10^{3}$ \\
\hline $\begin{array}{l}\text { CBR Serv E - } \\
\text { E delay (s) }\end{array}$ & $0.95 *$ & $0.98 *$ & $0.95 *$ & $0.95 *$ & $1.2 * 10^{-2}$ \\
\hline $\begin{array}{l}\text { CBR serv jitter } \\
\text { (s) }\end{array}$ & $1.67 * 1$ & $4 * 10^{-2}$ & $1.67 * 1$ & $1.67 * 1$ & $2.410^{-3}$ \\
\hline
\end{tabular}

Table 1: Comparison table for various scenarios using wireless access point technique

From the above table we observe that the throughput decreases with the number of moving nodes increasing. If the number of wireless access points increase, the numbers of handoffs will also increase. The end-to-end delay increases when the number of handoffs increases. Jitter also increases with number of handoffs. From scenario 2 and 4, we find that the packet loss exists due to the mobile nodes moving away from the base station. Mobile nodes get seamless connectivity.

\section{Conclusion}

Finally we conclude that using WAP techniques to provide handoff as well as connectivity in urban environment where signals are difficult to reach is a feasible alternative. Installation of expensive mini base stations can be avoided. In the future access points will be placed in all most every corner of an office building hence coverage would be better. Implementing access point based handoff can increase the Q.o.S

\section{References}

[1] "Development of dynamic clustering based handoff scheme, N.S.V.Shet, Prof.K.Chandrasekaran, Prof. K.C.Shet, NITK ,Surathakal, IETE conference , Icon RFW 09, Oct 09-11-2009, p.no 01-05, Bangalore.

[2] T. S. Rappaport, Wireless Communications. Prentice-Hall Inc., 1996.

[3] "Implementation of Handoff through wireless access techniques", N.S.V.Shet, Prof.K.Chandrasekaran, Prof. K.C.Shet,NITK ,Surathakal, Journal of telecommunications Vol 2, Issue 2, May 2010, p.no 143 146.

[4] IEEE. 802.11b/d3.0 Wireless LAN Medium Access Control (MAC) and Physical Layer (PHY) Specification, August 1999.

[5] Coexistence of Wireless Personal Area Networks with Other Wireless Devices Operating in Unlicensed Frequency Bands, Part 15.2.

[6] "ZigBee /IEEE 802.15.4 Summary", Sinem Coleri Ergen, September 2004.

[7] Wikipedia, http://en.wikipedia.org/wiki/Handoff. 
[8] Wikipedia, http://en.wikipedia.org/wiki/Wireless_access_point.

[9] Qualnet 4.5 Cellular Model Library.

[10] An Empirical Analysis of the IEEE 802.11 MAC Layer Handoff Process by Arunesh Mishra, Minho Shin, William Arbaugh

[11] "A survey of protocols for Wireless Sensor Networks, Rajesh Yadav, Shirshu Varma, N Malaviya"

[12]]Mobility Increases the Capacity of Ad Hoc Wireless Networks by Matthias Grossglauser and David N. C. Tse. IEEE/ACM TRANSACTIONS ON NETWORKING, VOL. 10, NO. 4, AUGUST 2002

[13] Qualnet 4.5 Wireless Model Library.

N.S.V.Shet is presently working in the Dept of E \& C, NITK, Surathkal as faculty of Engg. He is a M.E from IIT roorkee. He is pursuing his $\mathrm{PhD}$ at NITK, Surathkal His research interests are in the area of Wireless handoff and sensor networks. He has guided several Btech and Mtech projects. He was a visiting researcher at University Manchester Institute of science and technology, UK and Kagoshima University, Japan.

K.Chandra Sekaran is Professor in the Dept. of Computer Engg at NITK Surathkal. He has 22 years of teaching and academic research in the areas of networks, distributed computing, and over 95 publications in reputed journals / conferences.Incidently he is guiding N.S.V.Shet

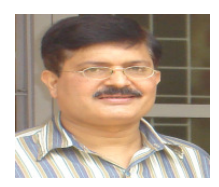

Prof K C Shet has 30 years of teaching experience. His interest are in Research, Design, Development and Consulting (including Counseling) in the area of Computers, Electronics, Software Engineering, and Information / Network Security.
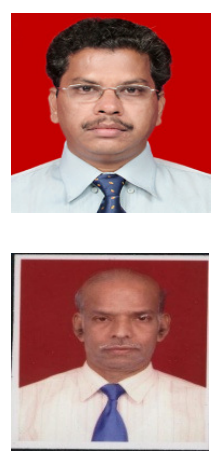